\title{
Factores de elección de carreras de Ciencias de la Comunicación en España: el peso de la ética
}

\author{
Joan Francesc FondeVILA GASCÓN \\ Universitat Abat Oliba CEU (UAO) \\ jfondevilag@uao.es \\ Josep Lluís Del Olmo ArriagA \\ Universitat Abat Oliba CEU (UAO) \\ jlolmo@uao.es
}

Recibido: 06/11/2012

Aceptado: 23/01/2013

\begin{abstract}
Resumen
Las carreras universitarias de Ciencias de la Comunicación cuentan en España con gran aceptación por parte de los estudiantes, lo que conduce al establecimiento de calificaciones elevadas para acceder a ellas. La actividad desarrollada en este ámbito implica un impacto y una trascendencia social elevados. En este contexto, es relevante el estudio de los factores éticos a la hora de elegir los estudios de Ciencias de la Comunicación. En este artículo recogemos los resultados de una investigación empírica en la que analizamos, junto a otros factores, la ética y los valores del estudiante a la hora de elegir estudios de Comunicación en la universidad. Se concluye que la influencia de la ética es mayor de lo previsto en las estrategias de elección de los estudiantes.
\end{abstract}

Palabras clave: ciencias de la comunicación, elección, España, ética, universidad

\section{Factors Career Choices of Communication Sciences in Spain: the Weight of Ethics}

\begin{abstract}
University courses in Communication Sciences in Spain have great acceptance by students, leading to the establishment of high qualifications to access them. Activity in this area has an impact and a high social significance. In this context, it is relevant to study the ethical factors when choosing studies of Communication Sciences. In this paper we present the results of an empirical study in which we analyzed, together with other factors, ethics and values of the student in choosing communication studies at the university. It is concluded that the influence of ethics is higher than expected in the strategies of student choice
\end{abstract}

Keywords: communication sciences, election, Spain, ethics, university

\section{Referencia normalizada}

FONDEVILA GASCÓN, Joan Francesc y DEL OLMO ARRIAGA, Josep Lluís (2013): "Factores de elección de carreras de Ciencias de la Comunicación en España: el peso de la ética". Estudios sobre el mensaje periodístico. Vol. 19, Núm. especial abril, págs.: 735-744. Madrid, Servicio de Publicaciones de la Universidad Complutense.

Sumario: 1. Introducción. 2. Metodología.3. Desarrollo. 4. Conclusiones. 5. Referencias bibliográficas

\section{Introducción}

Las carreras de Ciencias de la Comunicación son de las más solicitadas por los estudiantes en España. Como la actividad profesional que desarrollan los licenciados o graduados es de gran influencia social, es relevante analizar los factores de elección que llevan a los futuros universitarios a elegir carreras comunicativas en el contexto de la Sociedad de la Banda Ancha (Fondevila Gascón, 2012). 
Curiosamente, el proceso de elección de universidad, esencial para diseñar las políticas públicas educativas, no es objeto de estudio habitual en España. Los países anglosajones, liderados por Estados Unidos de América, Reino Unido, Canadá, Australia y Nueva Zelanda, han cultivado más este campo, aunque tampoco en demasía. Si el foco de estudio es la ética como factor de elección, entonces el terreno es yermo.

Hasta el momento, se han analizado variables concretas, como el sexo (Frotuny et altri, 1991), el impacto de la tecnología en la universidad y Secundaria (Fondevila Gascón y Carreras Alcalde, 2010; Fondevila Gascón et altri, 2012), el acceso a la universidad y los resultados del primer año, que dependen del éxito en la elección anterior (Corominas, 2001), el rendimiento académico en Secundaria, en la decisiva transición hacia la universidad (Fita et altri, 2004), los recursos económicos, culturales y sociales de las familias (Ball, 1993, Crozier, 1997), los mass media (Bowe et altri, 1994) o el acceso a la información, la anticipación, la negociación familiar o el cálculo (Veleda, 2002).

Como factores de elección se estudió la cercanía de la universidad, el aspecto y el estado de las instalaciones (West et altri, 1991), e incluso se propuso una estandarización para analizar el proceso de selección de universidad (Roszkowski y Spreat, 2010). Las características institucionales superan como factor de elección a los recursos interpersonales o de información utilizados por los estudiantes (Pampaloni, 2010).

En cuanto a la ética como factor concreto de elección, aparece en estudios parciales, aunque no como variable en la toma de decisiones de los estudiantes. La universidad venezolana generó una reflexión que incluyó el análisis de los valores, la universidad y la gestión (Mora, 2002). La solidaridad, la tolerancia y el concepto de cooperación guiaron el impacto ético en la universidad en América Latina (Soto, 2009). También se analizaron valores y habilidades con relación a contenidos específicos (Mosterio y Porto, 2000; Agejas et altri, 2007).

Una propuesta sobre las funciones universitarias relacionadas con las competencias profesionales de la enseñanza ética se sitúa en el Espacio Europeo de Educación Superior y analiza las actitudes éticas de los profesores con relación al plan de estudios (García López et altri, 2009).

La creciente competencia y la aparición de análisis comparativos generan interés por las herramientas utilizadas en la elección de la universidad. En España los polos son opuestos: universidades públicas y privadas (Fondevila Gascón et altri, 2011). Las públicas son un mercado de vendedores, al existir más demanda que oferta, lo que confiere ventaja competitiva, mientras que las privadas pertenecen a un mercado de compradores, en el que la oferta supera a la demanda (Fondevila Gascón y Del Olmo Arriaga, 2011).

\section{Metodología}

Desde el Grupo de Investigación sobre Periodismo Digital y Banda Ancha de la Universitat Abat Oliba CEU (UAO), en Barcelona, desarrollamos una encuesta en 2011 (metodología cuantitativa, fórmula probabilística aleatoria simple) a un universo representativo de los alumnos del primer curso de la Facultad de Ciencias Sociales (gra- 
dos de Periodismo, Publicidad y Relaciones Públicas, Derecho, Administración, Marketing y Administración de Empresas, Magisterio de Educación Infantil, Enseñanza de la Educación Primaria y Psicología) de la UAO. El objetivo era analizar empíricamente el proceso de elección de estudios de Comunicación en la universidad y el impacto de la ética y los valores.

El eje principal es el impacto de la ética, los valores y la religión en la toma de decisión. Se establecieron diez categorías generales para incluir 46 opciones de elección de universidad. Las categorías son las siguientes: conocimiento de la universidad; campus; coste y financiación; metodología; promoción e información; profesorado; servicios; bolsa de trabajo; estudiantes; y referencias. Las opciones Orientación humanística, ética e integral de la formación y Orientación religiosa de la formación se enmarcan en la categoría "Conocimiento de la universidad".

Al analizar la ética y la influencia de los valores y la religión como factores de decisión para elegir universidad, establecemos, en una metodología comparativa, los siguientes elementos: calidad de la enseñanza, ambiente de estudio, facilidad de acceso a la universidad, variedad de títulos oficiales ofrecidos, prestigio o reputación de la universidad, proyección internacional de la universidad, variedad de estudios especializados ofrecidos, orientación humanística, ética e integral de la formación y orientación religiosa de la formación. Analizamos estas posibilidades relacionadas con la intención de estudiar Comunicación en la universidad, el género, la demarcación o la existencia de un centro universitario en la ciudad de la familia.

La hipótesis de la investigación es que la ética y los valores (incluidos los religiosos) no son los principales factores para el alumnado a la hora de buscar información para analizar las diferentes alternativas en el proceso de elegir universidad.

Los factores tenidos en cuenta en la metodología elegida presentan antecedentes parciales en anteriores investigaciones. Así, el proceso de decisión para elegir una carrera depende de la disponibilidad, la flexibilidad y la variedad de la oferta, e incluso de un programa de actividades extra-académicas, externas o complementarias al programa principal de estudios. Para diversos autores, ese tipo de actividades es relevante para tomar la decisión de elegir una universidad o una carrera concretas (Kellaris y Kellaris, 1988; Buckley et altri, 1996; Witthuhn, 1997).

La opción de participar en actividades religiosas es mencionada como un factor de decisión para elegir universidad. En algunos estudios se prueba que el ambiente religioso es de relieve para los estudiantes en el momento de la elección (Roche et altri, 1987; Jonas, 1992). En Cataluña, pese a que no existen universidades de la Iglesia, la mayor parte de las privadas (UAO, UIC y URL) cuentan con raíces cristianas y ofrecen experiencias religiosas para los estudiantes que lo deseen.

En cuanto a una posible relación entre valores, religión y seguridad, se ha detectado influencia en la seguridad personal de los estudiantes (Mullet, 1985), más para los padres que para los estudiantes. Los autores posteriores que citan la seguridad como criterio se centran en varias poblaciones (Harris, 1994; Coccari y Javalgi, 1995) y en el ambiente religioso (Kellaris y Kellaris, 1988). Si bien algunos factores que afectan a las decisiones de los individuos son externos al sistema educativo, otros (actitudes psicológicas, coeficiente de inteligencia, ética y religión, número de hermanos, 
nivel educativo de los padres, costes directos y de oportunidad de la universidad, sistema de becas) influyen en las decisiones de los estudiantes.

\section{Desarrollo y resultados}

En una visión epidérmica y no reagrupadora de los resultados empíricos de la investigación, puede parecer que el factor ético, religioso y de valores no se encarama a las primeras posiciones en una tabla que incluye las 46 opciones sobre decisión. Así, en la Tabla 1, donde se muestran los factores de decisión y se incluyen los valores totales obtenidos para cada una de las proposiciones formuladas (ordenadas de mayor a menor influencia), los valores se ubican en una zona baja en la horquilla entre la calidad de la enseñanza $(4,17)$, como valor más representativo, y la orientación religiosa de la formación $(1,65)$, como atributo menos valorado por la muestra. El factor Orientación humanística, ética e integral de la formación $(2,85)$ se halla en una de las últimas posiciones. Los dos únicos factores que superan el nivel de mucha influencia (4) son la calidad de la enseñanza $(4,17)$ y la facilidad de acceso en transporte público $(4,02)$. El primer factor se enmarca en la categoría general Conocimiento de la universidad, y el segundo en Campus.

Tabla 1. Factores de decisión para elegir carreras de Comunicación (media: de 1 a 5).

Elaboración propia

\begin{tabular}{|c|c|c|c|}
\hline \multirow{2}{*}{$\begin{array}{l}\text { FACTORES DE DECISIÓN PARA } \\
\text { ELEGIR CENTRO }\end{array}$} & $\begin{array}{l}\text { TOTAL } \\
(2345)\end{array}$ & \multirow[t]{2}{*}{ GRUPO } & \multirow{2}{*}{$\begin{array}{c}\mathbf{N}^{\circ} \\
\text { orden }\end{array}$} \\
\hline & $\%$ & & \\
\hline Calidad de la enseñanza & 4.17 & Conocimiento de la universidad & 1 \\
\hline Facilidad de acceso en transporte público & 4.02 & Campus & 2 \\
\hline Coste anual de los estudios & 3.96 & Coste y financiación & 3 \\
\hline Ambiente de estudio & 3.88 & Conocimiento de la universidad & 4 \\
\hline Facilidades de financiación & 3.86 & Coste y financiación & 5 \\
\hline Facilidad de acceso a la universidad & 3.81 & Conocimiento de la universidad & 6 \\
\hline Orientación práctica de los estudios & 3.79 & Metodología & 7 \\
\hline Amabilidad en el trato del personal de la universidad & 3.74 & Promoción e información & 8 \\
\hline Accesibilidad del profesorado para los alumnos & 3.73 & Profesorado & 9 \\
\hline $\begin{array}{l}\text { Calidad en los servicios de asesoramiento y atención al } \\
\text { alumnado }\end{array}$ & 3.68 & Servicios & 10 \\
\hline Atractivo de las actividades ofertadas & 3.68 & Servicios & 11 \\
\hline Nivel de innovación tecnológica en la universidad & 3.66 & Servicios & 12 \\
\hline Atractivo de las instalaciones & 3.66 & Campus & 13 \\
\hline Experiencia profesional del profesorado & 3.63 & Profesorado & 14 \\
\hline Capacidades pedagógicas del profesorado & 3.58 & Profesorado & 15 \\
\hline Funcionamiento de la biblioteca & 3.57 & Servicios & 16 \\
\hline $\begin{array}{l}\text { Funcionamiento de servicios (cafetería, informática, co- } \\
\text { pistería...) }\end{array}$ & 3.51 & Servicios & 17 \\
\hline Variedad de titulaciones oficiales ofertadas & 3.50 & Conocimiento de la universidad & 18 \\
\hline Reputación de la universidad & 3.48 & Conocimiento de la universidad & 19 \\
\hline
\end{tabular}




\begin{tabular}{|c|c|c|c|}
\hline Proximidad de la universidad al domicilio habitual & 3.47 & Campus & 20 \\
\hline Proyección internacional de la universidad & 3.44 & Conocimiento de la universidad & 21 \\
\hline Eficacia de la bolsa de trabajo & 3.42 & Bolsa de trabajo & 22 \\
\hline Variedad de estudios especializados ofertados & 3.32 & Conocimiento de la universidad & 23 \\
\hline Nivel académico de los estudiantes & 3.32 & Estudiantes & 24 \\
\hline Proporción de aprobados por curso & 3.30 & Estudiantes & 25 \\
\hline Lengua de impartición de las clases & 3.26 & Metodología & 26 \\
\hline $\begin{array}{l}\text { Promoción e información directa a los alumnos poten- } \\
\text { ciales }\end{array}$ & 3.26 & Promoción e información & 27 \\
\hline Proporción de graduados insertados el primer año & 3.25 & Bolsa de trabajo & 28 \\
\hline Dificultad para acceder a una universidad pública & 3.25 & Referencias & 29 \\
\hline Reputación investigadora del profesorado & 3.23 & Profesorado & 30 \\
\hline Número de alumnos por clases & 3.13 & Metodología & 31 \\
\hline Recomendación de estudiantes universitarios & 3.07 & Referencias & 32 \\
\hline Posibilidad de vivir fuera del domicilio habitual & 3.03 & Campus & 33 \\
\hline Recomendación del profesor / tutor / orientador & 3.03 & Referencias & 34 \\
\hline $\begin{array}{l}\text { La universidad más próxima no imparte la titulación de- } \\
\text { seada }\end{array}$ & 2.99 & Campus & 35 \\
\hline Recomendación familiar & 2.96 & Referencias & 36 \\
\hline Atractivo de la oferta deportiva & 2.95 & Servicios & 37 \\
\hline Dificultad para acceder a una universidad privada & 2.95 & Referencias & 38 \\
\hline Recomendación de antiguos alumnos de la universidad & 2.91 & Referencias & 39 \\
\hline $\begin{array}{l}\text { Difusión de la universidad en los medios de comunica- } \\
\text { ción }\end{array}$ & 2.86 & Promoción e información & 40 \\
\hline $\begin{array}{l}\text { Orientación humanística, ética e integral de la forma- } \\
\text { ción }\end{array}$ & 2.85 & Conocimiento de la universidad & 41 \\
\hline Recomendación de amigos & 2.84 & Referencias & 42 \\
\hline Proximidad de la universidad al domicilio no habitual & 2.77 & Campus & 43 \\
\hline $\begin{array}{l}\text { Grado de vinculación de los ex-alumnos con la univer- } \\
\text { sidad }\end{array}$ & 2.49 & Estudiantes & 44 \\
\hline Decisión familiar & 2.49 & Referencias & 45 \\
\hline Orientación religiosa de la formación & 1.65 & Conocimiento de la universidad & 46 \\
\hline
\end{tabular}

En cuanto al factor de decisión de conocimiento de la universidad (Tabla 2), la orientación religiosa de la formación $(1,65)$ influye muy poco en la decisión, resultado que cuadra con la orientación laica de los centros públicos y la disminución progresiva de las creencias religiosas entre las generaciones jóvenes. No obstante, la orientación humanística, ética e integral de la formación consigue una puntuación más alta $(2,85)$. La suma de ambas categorías alcanza un 4,50 , lo que llevaría los valores y la religión a la cabeza de la tabla. 
Tabla 2. El conocimiento de la universidad como factor de decisión. Media de 1 a 5. Elaboración propia

\begin{tabular}{|l|c|c|c|c|c|c|c|}
\hline $\begin{array}{l}\text { FACTORES DE DECISIÓN PARA ELE- } \\
\text { GIR CENTRO: CONOCIMIENTO DE LA } \\
\text { UNIVERSIDAD }\end{array}$ & $\mathbf{( 2 3 4 5 )}$ & $\begin{array}{c}\text { Sí } \\
\mathbf{( 1 3 3 6 )}\end{array}$ & $\begin{array}{c}\text { Segura- } \\
\text { mente } \\
\text { sí (422) }\end{array}$ & $\begin{array}{c}\text { Aún no } \\
\text { lo sé } \\
\mathbf{( 2 2 8 )}\end{array}$ & $\begin{array}{c}\text { Segura- } \\
\text { mente } \\
\text { no (125) }\end{array}$ & $\begin{array}{c}\text { No } \\
\mathbf{( 2 2 3 )}\end{array}$ & $\begin{array}{c}\text { No } \\
\text { contesta } \\
\mathbf{( 1 2 )}\end{array}$ \\
\hline Calidad de la enseñanza & 4.17 & 4.27 & 4.07 & 3.98 & 3.95 & 3.95 & 4.21 \\
\hline Ambiente de estudio & 3.88 & 3.91 & 3.86 & 3.79 & 3.89 & 3.84 & 4.04 \\
\hline Facilidad de acceso a la universidad & 3.81 & 3.70 & 4.06 & 3.80 & 4.07 & 3.89 & 4.01 \\
\hline Variedad de titulaciones oficiales ofertadas & 3.50 & 3.46 & 3.54 & 3.58 & 3.65 & 3.52 & 2.75 \\
\hline Reputación de la universidad & 3.48 & 3.61 & 3.32 & 3.22 & 3.26 & 3.29 & 4.08 \\
\hline Proyección internacional de la universidad & 3.44 & 3.57 & 3.26 & 3.14 & 3.19 & 3.28 & 3.83 \\
\hline Variedad de estudios especializados ofertados & 3.32 & 3.34 & 3.28 & 3.32 & 3.19 & 3.34 & 3.16 \\
\hline $\begin{array}{l}\text { Orientación humanística, ética e integral de la } \\
\text { formación }\end{array}$ & 2.85 & 2.84 & 2.95 & 2.81 & 2.86 & 2.68 & 2.96 \\
\hline Orientación religiosa de la formación & 1.65 & 1.61 & 1.61 & 1.75 & 1.77 & 1.84 & 1.57 \\
\hline
\end{tabular}

Si observamos el género (Tabla 3), en el cómputo de las variables las mujeres otorgan a la ética y los valores un valor mayor que los hombres, salvo en la orientación religiosa de la formación $(1,61)$, cuya influencia es ligeramente inferior respecto a los hombres $(1,68)$. En contraste, en orientación humanística, ética e integral de la formación, las mujeres le asignan un 2,98, por 2,69 de los hombres. En ambos casos, hombres y mujeres, la suma de los valores éticos y religión conduciría a un hipotético liderato en la clasificación.

Tabla 3. Factores de decisión: el conocimiento de la universidad (por sexo).

Elaboración propia

\begin{tabular}{|l|c|c|c|}
\hline \multicolumn{1}{|c|}{ (Media de 1 a 5) } & \multirow{2}{*}{ Total } & \multicolumn{2}{c|}{ GÉNERO } \\
\cline { 3 - 4 } & & Hombre & Mujer \\
\hline $\begin{array}{l}\text { FACTORES DE DECISIÓN PARA ELEGIR CENTRO: } \\
\text { CONOCIMIENTO DE LA UNIVERSIDAD }\end{array}$ & $\mathbf{( 2 3 4 5 )}$ & $\mathbf{( 1 0 5 0 )}$ & $\mathbf{( 1 2 9 5 )}$ \\
\hline Calidad de la enseñanza & 4.17 & 4.01 & 4.30 \\
\hline Ambiente de estudio & 3.88 & 3.77 & 3.98 \\
\hline Facilidad de acceso a la universidad & 3.81 & 3.64 & 3.94 \\
\hline Variedad de titulaciones oficiales ofertadas & 3.50 & 3.33 & 3.63 \\
\hline Reputación de la universidad & 3.48 & 3.40 & 3.54 \\
\hline Proyección internacional de la universidad & 3.44 & 3.39 & 3.47 \\
\hline Variedad de estudios especializados ofertados & 3.32 & 3.19 & 3.42 \\
\hline Orientación humanística, ética e integral de la formación & 2.85 & 2.69 & 2.98 \\
\hline Orientación religiosa de la formación & 1.65 & 1.68 & 1.61 \\
\hline
\end{tabular}

En cuanto a la demarcación territorial (Tabla 4), a las cuatro demarcaciones provinciales de Cataluña (Barcelona, Tarragona, Girona y Lleida) hemos añadido una quinta demarcación, la ciudad de Barcelona, debido a su peso en el contexto catalán. En orientación humanística, ética e integral de la formación, la capital catalana es la que concede más puntuación, superando a Tarragona, Lleida, Barcelona y Girona. En orientación religiosa, Girona aventaja a Barcelona ciudad, Tarragona, Lleida y Bar- 
celona. No obstante, las cifras son similares entre las diversas demarcaciones y las diferencias no son significativas.

Tabla 4. Factores de decisión: el conocimiento de la universidad, por demarcación.

Elaboración propia

\begin{tabular}{|l|c|c|c|c|c|c|}
\hline \multirow{2}{*}{ (Media de 1 a 5) } & \multirow{2}{*}{ Total } & \multicolumn{5}{c|}{ DEMARCACIÓN } \\
\cline { 5 - 7 } & & $\begin{array}{c}\text { BCN } \\
\text { capital }\end{array}$ & $\begin{array}{c}\text { Barce- } \\
\text { lona }\end{array}$ & $\begin{array}{c}\text { Tarra- } \\
\text { gona }\end{array}$ & Girona & Lleida \\
\hline $\begin{array}{l}\text { FACTORES DE DECISIÓN PARA ELE- } \\
\text { GIR CENTRO: CONOCIMIENTO DE } \\
\text { LA UNIVERSIDAD }\end{array}$ & $\mathbf{( 2 3 4 5 )}$ & $\mathbf{( 6 6 1 )}$ & $\mathbf{( 1 1 3 5 )}$ & $\mathbf{( 2 2 1 )}$ & $\mathbf{( 1 9 2 )}$ & $\mathbf{( 1 3 6 )}$ \\
\hline Calidad de la enseñanza & 4.17 & 4.26 & 4.18 & 4.19 & 3.86 & 4.06 \\
\hline Ambiente de estudio & 3.88 & 3.86 & 3.93 & 3.85 & 3.76 & 3.85 \\
\hline Facilidad de acceso a la universidad & 3.81 & 3.74 & 3.82 & 4.07 & 3.62 & 3.90 \\
\hline Variedad de titulaciones oficiales ofertadas & 3.50 & 3.47 & 3.51 & 3.64 & 3.38 & 3.48 \\
\hline Reputación de la universidad & 3.48 & 3.64 & 3.48 & 3.32 & 3.19 & 3.37 \\
\hline Proyección internacional de la universidad & 3.44 & 3.52 & 3.46 & 3.46 & 3.12 & 3.26 \\
\hline Variedad de estudios especializados ofertados & 3.32 & 3.39 & 3.28 & 3.42 & 3.17 & 3.28 \\
\hline $\begin{array}{l}\text { Orientación humanística, ética e integral de la } \\
\text { formación }\end{array}$ & 2.85 & 2.94 & 2.82 & 2.90 & 2.68 & 2.85 \\
\hline Orientación religiosa de la formación & 1.65 & 1.72 & 1.58 & 1.66 & 1.77 & 1.63 \\
\hline
\end{tabular}

Sobre la existencia de un centro universitario en el municipio familiar (Tabla 5), los estudiantes consideran más los atributos cuando se goza de un centro en la población de origen. Los únicos factores ligeramente por debajo de la media en los estudiantes que disponen de centro universitario en su municipio son la facilidad de acceso a la universidad $(3,80)$, la variedad de títulos oficiales que se ofrecen $(3,44)$ y la orientación religiosa de la formación $(1,64)$. La suma de las orientaciones religiosas de la formación y la humanística, ética e integral u holística de la formación consigue de nuevo la primera posición.

Tabla 5. Los factores de decisión: conocimiento de la universidad si existe un centro universitario en la ciudad de origen. Elaboración propia

\begin{tabular}{|c|c|c|c|c|}
\hline \multirow{2}{*}{ (Media de 1 a 5) } & \multirow{2}{*}{ Total } & \multicolumn{3}{|c|}{$\begin{array}{l}\text { EXISTENCIA DE CENTRO UNI- } \\
\text { VERSITARIO EN EL MUNICIPIO }\end{array}$} \\
\hline & & No & Sí & $\mathbf{N} / \mathbf{C}$ \\
\hline $\begin{array}{l}\text { FACTORES DE DECISIÓN PARA ELEGIR CENTRO: } \\
\text { CONOCIMIENTO DE LA UNIVERSIDAD }\end{array}$ & (2345) & (1185) & (1109) & (51) \\
\hline Calidad de la enseñanza & 4.17 & 4.12 & 4.22 & 4.02 \\
\hline Ambiente de estudio & 3.88 & 3.86 & 3.91 & 3.85 \\
\hline Facilidad de acceso a la universidad & 3.81 & 3.82 & 3.80 & 3.63 \\
\hline Variedad de titulaciones oficiales ofertadas & 3.50 & 3.54 & 3.44 & 3.61 \\
\hline Reputación de la universidad & 3.48 & 3.41 & 3.56 & 3.40 \\
\hline Proyección internacional de la universidad & 3.44 & 3.35 & 3.52 & 3.48 \\
\hline Variedad de estudios especializados ofertados & 3.32 & 3.28 & 3.34 & 3.46 \\
\hline Orientación humanística, ética e integral de la formación & 2.85 & 2.78 & 2.91 & 3.03 \\
\hline Orientación religiosa de la formación & 1.65 & 1.65 & 1.64 & 1.84 \\
\hline
\end{tabular}


De esta forma, confirmamos la hipótesis de que la ética y los valores (religión incluida) no son factores prioritarios para el estudiante a la hora de tomar la decisión de elegir universidad, pero si sumamos ética, valores y religión, el conjunto superaría al resto de opciones.

\section{Conclusiones}

La ética, los valores y la religión se configuran como variables relevantes para seleccionar estudios de Ciencias de la Comunicación en la universidad. Aunque un análisis por separado de la orientación religiosa de la formación y la orientación humanística, ética e integral las sitúa en posiciones postreras como factor de decisión, la suma de ambas categorías arroja un resultado que supera al resto de opciones.

Podemos interpretar la posición moderada, individualmente, de la ética, los valores y la religión entre los factores para elegir la universidad a raíz de la orientación laica de los centros públicos y el retroceso en las creencias y la práctica religiosa de los jóvenes. No obstante, contra esa intuición, las cifras reflejan que no hay que desdeñar esas variables. El género, la demarcación o la existencia de un centro universitario en la población del estudiante no aportan diferencias significativas. Como propuestas de futuro, estudios longitudinales en Ciencias de la Comunicación y en otras áreas científicas y análisis sobre el cultivo de la ética en el ejercicio de la profesión pueden aportar datos sugerentes.

\section{Referencias bibliográficas}

AGEJAS, José Ángel; PARADA, José Luis; y OLIVER, Isaac (2007): "La enseñanza de la ética profesional en los estudios universitarios". Revista Complutense de Educación, vol. 18, n 2, pp. 67-86.

BALL, Stephen (1993): "Education Markets, Choice and Social Class: the market as a class strategy in the USA". British Journal of Sociology of Education, vol. 14, nº 1 .

BOWE, Richard; GEWIRTZ, Sharon; BALL, Stephen (1994): "Capturated by the Discourse? Issues and concerns in researching (parental choice)". British Journal of Sociology of Education, vol. 15, $\mathrm{n}^{\mathrm{o}} 1$.

BUCKLEY, Patrick G.; MAHAFFEY, Tom et altri (1996): "Educational Choices of Adult Learners". Journal of Marketing for Higher Education, vol. 7, n 3, pp. 1-15.

COCCARI, Ronald L.; JAVALGI, Rajshekhar G. (1995): “Analysis of students' needs in selecting a college or university in a changing environment". Journal of Marketing for Higher Education, vol. 6, n⿳⺈ 2, pp. 27-39.

COROMINAS, Eric (2001): "La transición de los estudios universitarios: abandono o cambio en el primer año de Universidad". Revista de Investigación Educativa, RIE, vol. $19, \mathrm{n}^{\mathrm{o}} 1$, pp. 127-152.

CROZIER, Gill (1997): "Empowering the powerful: a discussion of the interrelation of government policies and consumerism with social class factors and the impact of this upon parent interventions in their children's schooling". British Journal of Sociology of Education, vol. 18, $\mathrm{n}^{\mathrm{o}} 2$. 
FITA, Eva; RODRÍGUEZ, Sebastián; TORRADO, Mercedes (2004): "El rendimiento académico en la transición secundaria-universidad". Revista de Educación, n 334 , pp. 391-414.

FONDEVILA GASCÓN, Joan Francesc y CARRERAS ALCALDE, Marta (2010): "La tecnologia com a eina al servei de l'educació: una visió humanitzadora". Barcelona, Universitat Abat Oliba CEU.

FONDEVILA GASCÓN, Joan Francesc; DEL OLMO ARRIAGA, Josep Lluís; y SIERRA SÁNCHEZ, Javier (2011): "Universidades privadas, ¿un subsistema diferente?: cómo desarrollan los estudiantes sus preferencias hacia la universidad". Vivat Academia, $\mathrm{n}^{\circ}$ 114, marzo de 2011, pp. 1-19.

FONDEVILA GASCÓN, Joan Francesc y DEL OLMO ARRIAGA, Josep Lluís (2011): "La gestión de las preferencias hacia la universidad por parte de los estudiantes". Revista Electrónica Gestión de las Personas y Tecnología de la Facultad Tecnológica de la Universidad de Santiago de Chile USACH, julio de 2011, pp. 36-43.

FONDEVILA GASCÓN, Joan Francesc; CARRERAS ALCALDE, Marta; y DEL OLMO ARRIAGA, Josep Lluís (2012): "Impacto de las tecnologías de la información y la comunicación en la elección de Universidad: el caso de Internet y las redes sociales", en Edutec-E. Revista Electrónica de Tecnología Educativa, n 39, marzo 2012, 1-12.

FONDEVILA GASCÓN, Joan Francesc (2012): The Broadband Society in the World. Terrassa, CECABLE.

FROTUNY, Montserrat; PUJOL, María Rosario; y BORJA SOLÉ, María (1991): “Estudio diacrónico sobre la elección de las carreras superiores en función del sexo: propuestas de algunas medidas para conseguir la igualdad de oportunidades". Bordón. Revista de Pedagogía, vol. 43, n 1, pp. 91-104.

HARRIS, Tracy A. (1994): "Marketing for Black Alums". Journal of College Admissions, Summer, 5-11.

JONAS, Peter. M. (1992): "By the Seat of Your Pants". Journal of College Admissions. Winter, 12-15.

KELLARIS, James J.; KELLARIS, William K. L. (1988): "An Exploration of the Factors Influencing Students' College Choice Decision at a Small Private College". College and University, vol. 63, n 2, pp. 187-197.

MORA GARCÍA, José Pascual (2002): "Ética y universidad: reflexiones fenomenológicas para una filosofía de los valores en la universidad”. Acción Pedagógica, vol. $11, \mathrm{n}^{\circ} 2$, pp. 22-28.

MOSTERIO, María Josefa y PORTO, Ana María (2000): "Los motivos de elección de estudios en alumnos y alumnas de universidad". Innovación Educativa, vol. 10, pp. 121-132.

MULLET, Gary M. (1985): "Product Positioning Applied to Colleges: Methodology and Results". Journal of Professional Services Marketing, ${ }^{\circ}$ 1, pp. 97-133. 
PAMPALONI, Andrea M. (2010): "The influence of organizational image on college selection: what students seek in institutions of higher education". Journal of Marketing for Higher Education, vol. 20, nº 1, pp. 19-48.

ROCHE, Victoria F.; PETERS, L. L. et altri (1987): “Assessing the Attributes Which Influence Pharmacy School Selection". American Journal of Pharmaceutical Education, vol. 51, $\mathrm{n}^{\mathrm{o}} 3$, pp. 262-268.

ROSZKOWSKI, Michael J.; SPREAT, Scott (2010): "Weighing the difference: the validity of multiplicative and subtractive approaches to item weights in an instrument assessing college choice decisions". Journal of Marketing for Higher Education, vol. 20, n 2, pp. 209-239.

SOTO, Diana (2009): "El profesor universitario de América Latina: hacia una responsabilidad ética, científica y social". Revista Historia de la Educación Latinoamericana, $\mathrm{n}^{\circ} 13$, pp. 166-188.

VELEDA, Cecilia (2002): Estrategias individuales y familiares de elección de instituciones de educación superior. Buenos Aires, IIPE-UNESCO.

WEST, Anne; VARLAAM, Andreas; SCOTT, Georgia (1991): "Choice of high schools: pupils' perceptions". Educational Research, vol. 33, n 3, pp. 205-215.

WITTHUHN, Burton O. (1997): So You Want to Go to College? 50 Questions to Ponder. Port Orange, Fl, Cornesky \& Associates.

\section{Dr. Joan Francesc FONDEVILA GASCÓN}

Universitat Abat Oliba CEU (UAO)

Director del Departamento de Ciencias de la Comunicación y director del Centro de Estudios sobre el Cable (CECABLE)

jfondevilag@uao.es

\section{Dr. Josep Lluís DEL OLMO ARRIAGA}

Universitat Abat Oliba CEU (UAO).

Jefe de estudios de Marketing y Dirección Comercial

jlolmo@uao.es 